2008 and 2018, the greatest increase in risk of becoming looked after occurred in the most deprived local authorities, with the gap widening by 6 children per 100,000, per year (95\% CI 3 to 8 ). Ongoing analyses will determine how rising inequalities were distributed across age groups and children's category of need upon entering care. Fixed effects models will be used to assess the relationship between changes in determinants of children entering care, such as child poverty and expenditure on preventative services, and changes in risk of becoming looked after.

Conclusion This study provides evidence that the dramatic rise in children taken into care has been greater in the poorest areas of the country. Policies that address the determinants of children entering care at local area level are important for tackling the inequalities picture, and the rise in LAC. A limitation of this analysis relates to the use of 2015 income deprivation scores, which may not accurately reflect the relative status of local authorities across all years.

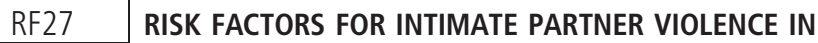 ASYLUM SEEKING AND REFUGEE POPULATIONS: A SYSTEMATIC REVIEW}

${ }^{1} \mathrm{R}$ El-Moslemany*, 'L Mellon, 'L Tully, ${ }^{2} \mathrm{~S}$ McConkey. ${ }^{1}$ Psychology, Royal College of Surgeons in Ireland, Dublin, Ireland; ${ }^{2}$ International Health and Tropical Medicine, Royal College of Surgeons in Ireland, Dublin, Ireland

\subsection{6/jech-2019-SSMabstracts. 142}

Background Intimate partner violence (IPV) is a pressing public health concern with well-documented evidence of adverse health consequences including mortality, physical, psychological and sexual harm. The specific experience of the asylum seeking and refugee populations includes exposure to pre-migration, migration and post-migration risk factors for adverse events. This migration history, along with factors such as differences in cultural values and norms may make these populations especially vulnerable to IPV. The aim and objective of this review was to identify risk factors for IPV in the asylum seeking and refugee populations by synthesising and critiquing the literature on this topic.

Methods We identified 7,219 records by searching the PubMed, Web of Science, SCOPUS, Global Health, Embase, PsycINFO, Westlaw and SSRN electronic databases. We searched grey literature databases, agency websites and repositories. We critically appraised and extracted data using the JBI SUMARI tool. We ranked evidence quality using the GRADE approach. Meta-analysis was not possible due to heterogeneity. We took a framework analysis approach to narrative synthesis i.e. familiarisation, identifying a thematic framework, indexing, charting, mapping and interpretation. We considered any risk factor as the exposure and IPV as the outcome. We separated asylum seekers and refugees for subgroup analysis. We registered the review with PROSPERO and adhered to the PRISMA reporting criteria.

Results This review is ongoing. Searching resulted in 7,220 records. There were 4,693 records after we removed duplicates. We screened 4,693 titles and abstracts, selecting 52 studies for full-text assessment against explicit eligibility criteria. We excluded 28 studies with reasons for exclusion. We included 24 studies in the analysis. Twenty studies had a cross-sectional design, three were prevalence studies and one a cohort study. Political violence, conflict victimization, attitudes to IPV, previous experience of IPV and education emerged as categories of risk factors that may be associated with IPV. $96 \%$ of studies had a cross-sectional design, therefore temporality and direction of associations were difficult to determine. Discussion There is a large gap in research and policy-relevant evidence in this area. This review is important because it may be the first systematic review on this particular topic. The challenges for this study include inconsistent terminology, absent definitions, validity and reliability of measures of risk factors and IPV, self-report of IPV. This review may have implications for policy on safeguarding residents in reception centres and camps, screening for and reporting of IPV in clinical practice and future research.

\section{RF28 AREA- AND INDIVIDUAL-BASED MEASURES OF SOCIOECONOMIC CIRCUMSTANCES AND ADHD PRESCRIPTION UPTAKE AMONG YOUNG CHILDREN IN SCOTLAND: A POPULATION-BASED REGISTER STUDY}

${ }^{1} \mathrm{PM}$ Henery*, 'SV Katikireddi, ${ }^{2} \mathrm{R}$ Wood, ${ }^{1} \mathrm{R}$ Dundas, ${ }^{1} \mathrm{~A}$ Leyland, ${ }^{1} \mathrm{~A}$ Pearce. ${ }^{1} \mathrm{MRC} / \mathrm{CSO}$ Social and Public Health Sciences Unit, University of Glasgow, Glasgow, UK; ${ }^{2}$ Information Services Division, NHS National Services Scotland, Edinburgh, UK

\subsection{6/jech-2019-SSMabstracts. 143}

Background Attention-deficit hyperactivity disorder (ADHD) in childhood can have adverse effects on mental health, learning, and employment opportunities. There is evidence of a relationship between socioeconomic disadvantage and likelihood of ADHD in childhood; however, most studies use area-level measures which may underestimate inequality compared to individual/family-based measures. This study aimed assess whether area-level and individual-level measures of social disadvantage were predictive of child ADHD via dispensed prescriptions.

Methods We used birth data for all children born in Scotland 2010-2012 $(n=195,419)$ linked to Prescription Information System up to March 2018. Two measures of socioeconomic circumstances (SECs) at the child's birth were used: Scottish Index of Multiple Deprivation (SIMD) (area-based), and four class measure of the NS-SEC of the mother (individual-based). Prescription use was defined as a record of any dispensed prescription for ADHD up until March 2018 (median age 6). We used binary logistic regression to estimate risk ratios (RRs) for prescription uptake by each SEC measure before and after adjusting for covariates/confounders (sex, age of child as of March 2018, number of births in pregnancy, mother's age at first live birth, mother's birth country, relationship status of parents).

Results Prescription use varied by area deprivation $(0.58 \%$ of children born in the most deprived SIMD decile compared to $0.14 \%$ in the least deprived) and social class $(0.62 \%$ for children born to mothers in the unemployed/other social class compared to $0.16 \%$ in the managerial/professional social class). The strength of association narrowed slightly after adjustment for confounders/covariates; the fully adjusted RR for prescription use was 2.14 (95\% C.I.: 1.33-3.44) in the most compared to least deprived SIMD decile and 2.32 (95\% C.I.: 1.78-3.04) for children born to mothers who were unemployed/other compared to managerial/professional. After mutual adjustment for both SEC measures, the effect of SIMD was reduced whilst that of mother's social class remained consistent.

Conclusion Both area-level and family-level deprivation at birth are associated with increased prescription use among 
young children in Scotland, suggesting disadvantaged SEC are associated with higher prescription use. Inequalities in ADHD prescriptions across childhood may not be fully captured since prevalence increases with age (and our data only follow children up to the maximum age of 8). Prescription data may underestimate prevalence of $\mathrm{ADHD}$ as not all children with ADHD symptoms will be diagnosed and/or prescribed (and this may vary by SEC). Future analyses will explore this using data from child health checks.

\section{RF29 VIOLENCE AGAINST PEOPLE WITH DISABILITES: VARIATIONS BY IMPAIRMENT TYPE, GENDER AND SOCIO-ECONOMIC STATUS IN AN AUSTRALIAN POPULATION-BASED STUDY}

'L Krnjacki*, ${ }^{2} E$ Emerson, ${ }^{2} \mathrm{G}$ Llewellyn, 'Z Aitken, 'A Kavanagh. ' Centre for Health Equity, The University of Melbourne, Melbourne, Australia; ${ }^{2}$ Centre for Disability Research and Policy, The University of Sydney, Sydney, Australia

\subsection{6/jech-2019-SSMabstracts. 144}

Background Recent international meta-analyses show that children and adults with disabilities are more likely to experience interpersonal violence than those without disabilities. People with disabilities are a heterogeneous group, and further work is required to determine the variations in the experience of violence by impairment type. It is also important to assess the extent to which any between-group differences may be explained by socio-economic situation.

Methods We analysed the 2015 Australian Bureau of Statistics Survey on Personal Safety of more than 21,000 adults. We used population-weighted, age-adjusted, logistic regression to estimate the odds of violence in the last 12 months by impairment type and gender. We stratified by five forms of violence (physical, sexual and intimate partner, emotional abuse and stalking/harassment) and for specific impairment types (physical, intellectual, psychological and sensory). We also investigated potential effect modification by poverty status.

Results Results for women showed that those with sensory and physical impairments fared the best, with a two-fold increase in odds across all five forms of violence. Women with intellectual and psychological impairments fared worse for all forms of violence. There was a three-fold increase in the odds for emotional abuse and stalking/harassment. There was an even greater increase in the odds for sexual, physical and partner violence. Women with intellectual impairments had between a five and seven-fold increase in the odds of these forms of violence, while women with psychological impairments had been a four and six-fold increase. All results were statistically significant at $\mathrm{p}=0.05$.

Results for men showed much less variation by impairment type. There was a statistically significant two-fold increase in odds of physical violence for those with sensory, intellectual and psychological impairments. There were no significant differences between men with impairments compared to men without disabilities for sexual violence, partner violence and emotional abuse. There was evidence that stalking/harassment was worse for men with psychological impairments.
Some of these associations were moderated by poverty status, with the increased odds of exposure to violence among people with disabilities being greater for people living in poverty.

Conclusion These results show there are important variations in the experience of violence for people with disability depending on their impairment type, gender and socio-economic status. There is a clear need to develop interventions that are targeted to the particular circumstances and needs of these high-risk groups.

\section{RF30 RESIDENTIAL AREA DEPRIVATION PREDICTS SUBSEQUENT HOSPITAL ADMISSION IN A BRITISH POPULATION INDEPENDENTLY OF SOCIAL CLASS AND EDUCATION STATUS: THE EPIC-NORFOLK COHORT}

${ }^{1}$ RN Luben*, 'SA Hayat, ${ }^{2} \mathrm{NJ}$ Wareham, ${ }^{1} \mathrm{PD}$ Pharoah, ${ }^{1} \mathrm{KT}$ Khaw. ${ }^{1}$ Public Health and Primary Care, University of Cambridge, Cambridge, UK; ${ }^{2}$ MRC Epidemiology Unit, University of Cambridge, Cambridge, UK

\subsection{6/jech-2019-SSMabstracts. 145}

Background Low social economic position is linked to higher rates of mortality and morbidity. Socioeconomic factors are reported to predict admission to hospital for many conditions. It is less clear if residential area deprivation index predicts hospital usage independently of individual social class and lifestyle factors.

Methods We examined the relationship between residential Townsend's Area Deprivation Index and subsequent admissions to hospital and time spent in hospital for 11,214 men and 13,763 women aged 40-79 years in the general population.

Participants from the EPIC-Norfolk prospective populationbased study were followed for nineteen years (1999-2018) using record linkage. Townsend's Index from the small area measurements taken at the 1991 UK census was linked to individuals in the EPIC-Norfolk cohort using their postal code.

Results Compared to those with residential Townsend Area Deprivation Index lower than the average for England and Wales, those with a higher than average deprivation index had a higher likelihood of spending more than twenty days in hospital multivariable adjusted odds ratio (OR) 1.18 (95\% confidence interval (CI) 1.07-1.29) and having 7 or more admissions OR 1.11 (95\% CI 1.02-1.22) after adjustment for age, sex, smoking status, education, social class, body mass index and prevalent diseases. Those with manual social class were at greater risk of hospitalisation compared to those with a non-manual social class when living in an area with higher deprivation index $(p$ Interaction $=0.025)$. Similarly, those with a lower education level living in a more deprived area had a higher risk of hospitalisation ( $\mathrm{p}$ Interaction $=0.020$ ) when compared with those with a higher education level.

Conclusion Residential area deprivation predicts future hospitalisations - time spent in hospital and number of admissions independently of individual social class and education level and other behavioural factors. There are significant interactions such that residential area deprivation has greater impact in those with low education level or manual social class. 